Letter to the Editor

\title{
Does this robot have a mind? Schizophrenia patients' mind perception toward humanoid robots
}

\author{
Stéphane Raffard ${ }^{\mathrm{a}, \mathrm{b}, *}$, Catherine Bortolon ${ }^{\mathrm{a}, \mathrm{b}}$, Laura Cohen ${ }^{\mathrm{c}}$, Mahdi Khoramshahic ${ }^{\mathrm{c}}$, Robin N. Salesse ${ }^{\mathrm{d}}$, \\ Aude Billard ${ }^{\mathrm{c}}$, Delphine Capdevielle $\mathrm{e}^{\mathrm{b}, \mathrm{e}}$ \\ a Epsylon Laboratory Dynamic of Human Abilities \& Health Behaviors, University of Montpellier 3, Montpellier, France \\ ${ }^{\mathrm{b}}$ University Department of Adult Psychiatry, Hôpital de la Colombière, CHRU Montpellier, Montpellier University, Montpellier, France \\ ${ }^{c}$ Learning Algorithms and Systems Laboratory, School of Engineering, EPFL, Lausanne, Switzerland \\ d EuroMov, Montpellier University, 700 Avenue du Pic Saint-Loup, 34090 Montpellier, France \\ e INSERM U-1061, Montpellier, France
}

\section{Dear Editors}

The introduction of social robots in our homes has become a reality. Numerous companies are currently developing robots that are accessible to everyone in order to help people to live easier and safer lives. At the same time, robotic therapies for the rehabilitation of social deficits have recently emerged in the field of autism disorder and dementia (Scassellati et al., 2012). Technological advances have enabled robots to fulfill a variety of human-like functions, as well as to support improvements in social skills in clinical populations. One important question in the field of socially assistive or home-robots concerns the variables influencing both the use of robots and the quality of human-robot interaction. Converging evidence suggests that successful interactions with others require recognizing which entities have minds and which do not, a process known as "mind perception" (Gray et al., 2007). Similarly, in the field of social robotics, mind perception toward robots is an important predictor of the quality of human-robot interactions and human behaviors toward robots (Boradbent, 2017). However, how individuals with schizophrenia ascribe a mind to robots and more particularly humanoid robots has never been yet explored despite the fact that socially assistive robots could be of particular interest for the rehabilitation of negative symptoms such as apathy or social deficits (Narita et al., 2016) (Table 1).

* Corresponding author at: Laboratory Epsylon, EA 4556, Montpellier University 3, 1 University Department of Adult Psychiatry, 39 Avenue Charles Flahault, 34295 Montpellier Cedex 5, France.

Email address: s-raffard@chu-montpellier.fr (S. Raffard)
The main aim of the present study was to explore how schizophrenia patients attribute mind to a humanoid robot compared to healthy individuals.

22 schizophrenia patients and 22 age, premorbid IQ, educational level and gender-matched healthy individuals were recruited for this study. Patients completed the Positive and Negative Syndrome Scale (PANSS). Besides the clinical assessments, all participants filled out the Mind Perception Questionnaire (MPQ; Gray et al., 2007) and the f-NART (Mackinnon \& Mulligan, 2005) after the end of the collaborative task (see description below).

In this experiment (see Cohen et al., 2017 for a full description of the experiment), we used the iCub, a $1.20 \mathrm{~m}$ tall humanoid robot (see Fig. 1, Supplementary data), specifically designed to support research in embodied artificial intelligence. The quality of the collaboration between the robot and the participant was evaluated in the context of an imitation task called the mirror game (Noy et al., 2011) whereby two players mirror each other's hand motions (see Fig. 2, Supplementary data).

As psychotic symptoms outcome we used the five factors of the PANSS proposed by van der Gaag et al. (2006), subdivided into the factors: positive symptoms, negative symptoms, disorganization, excitement, and emotional distress.

As mind perception outcome we used the MPQ. The MPQ evaluates how individuals perceive living and non-living things in terms of Experience (e.g. How much is the agent capable of experiencing physical or emotional pleasure?) and Agency (e.g. How much is the agent capable of remembering things?). 
Table 1

Mean (M) and Standard Deviation (SD) or Median and Min-Max scores of schizophrenia patients and healthy controls for socio-demographic, mindreading and clinical characteristics.

\begin{tabular}{|c|c|c|c|c|c|c|c|c|c|c|c|c|}
\hline & \multirow{2}{*}{$\begin{array}{l}\text { Healthy controls } \\
\mathrm{M}\end{array}$} & \multicolumn{3}{|c|}{ Schizophrenia patients } & \multicolumn{6}{|c|}{ Statistics } & \multirow[b]{2}{*}{ d } & \\
\hline & & SD & M & SD & & $\mathrm{t}$ & & $\mathrm{df}$ & $p$ & & & \\
\hline Age & 29.05 & 7.23 & 29.68 & 6.03 & & & 317 & 42 & 0.75 & 3 & 0.09 & \\
\hline \multirow[t]{3}{*}{ Fnart } & 105.52 & 6.27 & 105.84 & 10.56 & & & 122 & 42 & 0.90 & & 0.03 & \\
\hline & & & & & \multicolumn{2}{|c|}{ Healthy controls } & \multicolumn{2}{|c|}{ Schizophrenia patients } & Stati & stics & & \\
\hline & & & & & Median & Min-Max & Median & Min-Max & $\mathrm{U}$ & Z & $p$ & $d$ \\
\hline \multicolumn{13}{|c|}{ Mind perception } \\
\hline Agen & & & & & 1.70 & $1-3.20$ & 2.60 & $1-4.80$ & 152 & -2.125 & 0.034 & 0.67 \\
\hline Expe & & & & & 1.40 & $1-2.20$ & 1.60 & $1-4.60$ & 201 & -0.975 & 0.330 & 0.29 \\
\hline \multicolumn{13}{|c|}{ Clinical characteristics } \\
\hline Positi & toms & & & & & & 8.5 & $6-18$ & & & & \\
\hline Nega & toms & & & & & & 9.5 & $7-21$ & 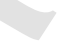 & & & \\
\hline Disor & & & & & & & 11 & $9-21$ & & & & \\
\hline Excit & & & & & & & 4 & $4-9$ & & & & \\
\hline Emot & tress & & & & & & 6.5 & $4-17$ & & & & \\
\hline Daily & mazine & (eq) $d r$ & (mg/day & & & & 250 & $125-500$ & & & & \\
\hline Durat & ness (ye & & & & & & 7 & $0.5-30$ & & & & \\
\hline
\end{tabular}

Using Mann-Whitney $U$ tests, significant differences were found on the mind perception questionnaire. Schizophrenia patients had higher scores than controls on agency dimension only $(\mathrm{U}=152$, $p=0.034, \mathrm{~d}=0,67)$. Regarding psychotic dimensions, a significant negative correlation was found between Experience and Emotional Distress $(r=-0.443, p=0.039)$. No other correlations were found between other clinical and demographic variables and mind perception dimensions.

The use of robotics technology in mental health care is nascent and requires exploratory studies to investigate the potential of emerging technologies. Our results showed that schizophrenia patients had an increased tendency compared to healthy controls to ascribe mental capacities to a humanoid robot for agency-related capacities (the capacity to plan and act). These findings are partially in line with the study of Gray et al. (2011) who showed that increased levels of schizotypy symptoms were linked to increased perceptions of agency and experience in entities generally thought to lack minds like trees and the dead. They also indicate that a basic function of social cognition, that is mind perception, is distorted in schizophrenia and could underlie some higher social cognitive processes that have been found to be repeatedly impaired in schizophrenia patients. From a humaninteraction perspective, it has been suggested that people who attributed more agency to robots may be less likely to use the robot (Broadbent et al., 2013). This tendency for schizophrenia patients to over attribute agency to humanoid robots could thus negatively influence the use of robots in this clinical population.

Finally, the dimension emotional distress was negatively associated with the dimension Experience. As the PANSS was completed before the interaction with $\mathrm{iCub}$, this finding indicates that higher emotional distress lead patients to ascribe to a humanoid robot less agency and cause an objectification of the humanoid robot.

In conclusion, if there is no doubt that robots could assist schizophrenia patients in a variety of their living daily tasks, mind perception and other social cognition deficits could negatively impact their use and efficiency.

Supplementary data to this article can be found online at https:// doi.org/10.1016/j.schres.2017.11.034.

\section{Conflict of interest statement}

The authors declare that they have no competing financial or other interests that might be perceived to influence the results and discussion reported in this paper.

\section{Contributors}

Stéphane Raffard, Aude Billard, Laura Cohen, Catherine Bortolon, Robin Salesse, Mahdi Khoramshahi, and Delphine Capdevielle contributed to the study design. Catherine Bortolon, recruited and assessed the patients. Catherine Bortolon and Laura Cohen performed the statistical analysis. Stéphane Raffard wrote the first draft. Stephane Raffard and Catherine Bortolon prepared the final manuscript, with feedback from the other authors.

\section{Role of funding source}

Authors are funded by the European project AlterEgo (FP7-ICT2011-9 “Cognitive Systems and Robotics" \# 600610).

\section{Uncited references}

Stafford et al., 2014

Walters et al., 2008

\section{Acknowledgments}

We express our gratitude to all the participants who participated in this research and thereby made it possible.

\section{References}

Broadbent, E., Kumar, V., Li, X., Sollers 3rd, J., Stafford, R.Q., MacDonald, B.A., Wegner, D.M., 2013. Robots with display screens: a robot with a more humanlike face display is perceived to have more mind and a better personality. PLoS One 8 (8), e72589.

Cohen, L., Khoramshahi, M., Salesse, R.N., Bortolon, C., Słowinski, P., Zhai, C., Tsaneva-Atanasova, K., Di Bernardo, M., Capdevielle, D., Marin, L., Schmidt, R.C., Bardy, B.G., Billard, A., Raffard, S., 2017. From smile to synchrony: the influence of social feedback and mind perception during a cooperative humanrobot task in schizophrenia. Sci. Rep. 7 (1), 15023. 
Gray, H.M., Gray, K., Wegner, D.M., 2007. Dimensions of mind perception. Science 315 (5812), 619.

Gray, K., Jenkins, A.C., Heberlein, A.S., Wegner, D.M., 2011. Distortions of mind perception in psychopathology. Proc. Natl. Acad. Sci. U. S. A. 108 (2), 477-479.

Narita, S., Ohtani, N., Waga, C., Ohta, M., Ishigooka, J., Iwahashi, K., 2016. A pettype robot Artificial Intelligence Robot-assisted therapy for a patient with schizophrenia. Asia Pac. Psychiat. 8 (4), 312-313.

Noy, L., Dekel, E., Alon, U., 2011. The mirror game as a paradigm for studying the dynamics of two people improvising motion together. Proc. Natl. Acad. Sci. 108, 20947-20952.

Scassellati, B., Admoni, H., Matarić, M., 2012. Robots for use in autism research Annu. Rev. Biomed. Eng. 14, 275-294.
Stafford, R.Q., MacDonald, B.A., Jayawardena, C., Wegner, D.M., Broadbent, E., 2014. Does the robot have a mind? Mind perception and attitudes towards robots predict use of an eldercare robot. Int. J. Soc. Robot. 6, 17-32.

van der Gaag, M., Hoffman, T., Remijsen, M., Hijman, R., de Haan, L., van Meijel, B., van Harten, P.N., Valmaggia, L., de Hert, M., Cuijpers, A., Wiersma, D. 2006. The five-factor model of the Positive and Negative Syndrome Scale II: a ten-fold cross-validation of a revised model. Schizophr. Res. 85, 280-287.

Walters, L.W., Syrdal, C.S., Dautenhahn, K., Boekhorst, R., Koay, K.L., 2008 Avoiding the uncanny valley: robot appearance, personality and consistency of behavior in an attention-seeking home scenario for a robot companion?. Auton. Robot. 24, 159-178. 